\title{
Singularities of Mean Curvature Flow and Flow with Surgeries
}

\author{
Carlo Sinestrari
}

\begin{abstract}
We collect in this paper several results on the formation of singularities in the mean curvature flow of hypersurfaces in euclidean space, under various kinds of convexity assumptions. We include some recent estimates for the flow of 2-convex surfaces, i.e. the surfaces where the sum of the two smallest principal curvatures is positive everywhere. Such results enable the construction of a flow with surgeries for these surfaces similar to the one introduced by Hamilton and Perelman for the Ricci flow. The topological applications of the construction are also described.
\end{abstract}

\section{Introduction}

Let $F_{0}: \mathcal{M} \rightarrow \mathbb{R}^{n+1}$ be a smooth immersion of an $n$-dimensional hypersurface in Euclidean space, $n \geq 1$. The evolution of $\mathcal{M}_{0}=F_{0}(\mathcal{M})$ by mean curvature flow is a one-parameter family of smooth immersions $F: \mathcal{M} \times\left[0, T\left[\rightarrow \mathbb{R}^{n+1}\right.\right.$ satisfying

$$
\begin{gathered}
\frac{\partial F}{\partial t}(p, t)=-H(p, t) \nu(p, t), \quad p \in \mathcal{M}, t \geq 0, \\
F(\cdot, 0)=F_{0},
\end{gathered}
$$

where $H(p, t)$ and $\nu(p, t)$ are the mean curvature and the outer normal respectively at the point $F(p, t)$ of the surface $\mathcal{M}_{t}=F(\cdot, t)(\mathcal{M})$.

This evolution has been studied by many authors in the last decades. It occurs in some physical models describing interface evolution, and also in the singular limit of some reaction diffusion equations. It can be checked that problem (1.1)-(1.2) is parabolic and possesses a unique solution locally in time. In general, however, global existence cannot be expected, because the curvature can become unbounded in finite time. This is always the case, for instance, if the surface is closed. Intuitively, certain parts of the surface, or the whole surface, shrink with the flow and develop singularities. 
It is natural to look for a generalized definition of the flow which allows to continue the evolution after the formation of singularities. The first approach was introduced by Brakke [7], who gave a definition of weak solutions using notions from geometric measure theory. Several other notions have been introduced in the following. Among others, we recall the one based on the level set method which was adopted independently by Chen, Giga and Goto [8] and by Evans and Spruck [14].

A further reason of interest for the mean curvature flow came from Hamilton's paper on the Ricci flow [19]. The Ricci flow is the evolution of the metric of an abstract Riemannian manifold governed by the equation

$$
\frac{\partial g_{i j}}{\partial t}=-2 R_{i j}
$$

where $R_{i j}$ is the Ricci tensor associated with the metric. Like the mean curvature flow, the Ricci flow is a parabolic problem where singularities can appear in finite time. Hamilton's result in [19] was that any initial metric on a closed three-dimensional manifold with positive Ricci curvature evolving by Ricci flow converges, up to a suitable rescaling, to a metric of constant curvature. This implies that any three-dimensional Riemannian manifold with positive Ricci curvature is diffeomorphic to a quotient of the standard sphere $S^{3}$. Such an approach had an antecedent in the work of Eels and Sampson [13], who had used the heat flow to deform a map between two fixed Riemannian manifolds. Hamilton's result, which was soon followed by similar ones for dimensions other than three, showed that the study of geometric flows could provide new important results.

In [24] Huisken proved a theorem for the mean curvature flow which had strong analogies with Hamilton's one for the Ricci flow. He showed that any closed convex surface shrinks to a point in finite time and converges, after rescaling, to a round sphere. Also, it became clear that the two flows have many similarities. The basic examples of formation of singularities (e.g., the sphere, the neckpinch) are analogous for the two flow. In both flows some important geometric properties remain invariant. For example, positive curvature operator and positive scalar curvature are preserved under Ricci flow, while convexity and positive mean curvature are preserved under mean curvature flow. All these results follow easily by the maximum principle applied to the evolution equation for the corresponding scalar or tensor quantity. For both flows the formation of singularities induces in some case the convergence to a canonical structure (constant curvature). It should be pointed out that there are no precise connections between Ricci flow and mean curvature flow, that is, there is no way to transform one flow into the other. Also, the proofs in [19] and [24] differ in many substantial steps. Throughout the paper, we will remark further the analogies and the differences between the two flows.

In the last years, the Ricci flow has become well known in the mathematical community after Perelman's proof $[\mathbf{3 4}, \mathbf{3 5}, \mathbf{3 6}]$ of Thurston's 
geometrization conjecture, a result which gives a complete classification of closed three-dimensional manifolds, and includes the celebrated Poincaré conjecture. Perelman's proof accomplishes a program started by Hamilton shortly after his above mentioned paper, a program aimed to prove the conjecture using the Ricci flow. A central tool in the proof is the construction of a flow after singularities by means of a surgery procedure: when a singularity occurs, the parts of the manifold with larger curvature are removed and are replaced with more regular ones. The surgery is performed in such a way that one keeps track of the possible changes of the topology. Hamilton conjectured that it was possible to define a flow where, after a finite number of surgeries, the remaining components converge to canonical structures, allowing to classify the possible topologies of the initial manifold. The hardest part in this analysis is to derive suitable estimates for the flow which give a description of the singular profiles good enough to do the surgeries, and which allow to prove that the remaining components eventually converge to the desired canonical structure. Hamilton carried out his program in the simpler case the Ricci flow of four-dimensional manifolds with positive isotropic curvature in [23]. However, the case of a general three-manifold needed for geometrization presented some further major difficulties. These were successfully solved by Perelman, who found some new estimates and introduced new techniques which allowed the conjectures to be proven.

The aim of the present paper is to give a survey of some recent results in collaboration with $\mathrm{G}$. Huisken $[\mathbf{2 7}, \mathbf{2 8}, \mathbf{2 9}$ ], which can be regarded as the counterpart for the mean curvature flow of Hamilton's approach to the Ricci flow. Namely, we define a mean curvature flow with surgeries for hypersurfaces satisfying a suitable curvature restriction, which we explain below. Like in the three-dimensional Ricci flow, our flow with surgeries gives rise after a finite number of steps to components which have a canonical structure, and allows a classification of the possible topologies of the initial manifold. The applications of our construction are not as far-reaching as the ones of Ricci flow, but they show that also mean curvature flow can be used to derive nontrivial topological consequences. We hope that in the future the technique can be extended to more general classes of surfaces to obtain a wider range of applications.

Let us briefly describe our surgery procedure. Following Hamilton [23], we call a neck a subset of our surface which is close, up to rescaling, to a portion of the standard cylinder $S^{n-1} \times \mathbb{R}$ in a suitable topology. Our surgery consists of removing a neck and filling smoothly the two holes with convex regions diffeomorphic to disks. The surface changes its topology in a controlled way, because the surgery acts like the inverse of a connected sum. The aim is to perform surgeries in such a way that the parts of the surface with largest curvature are removed and the flow can be continued for a longer time. To this purpose, one needs to show that it is possible to find a neck whenever a singularity is formed (except for those cases where we can already describe the topology of the whole manifold) and that the curvature 
decreases after the neck is removed. In order to prove these properties, it is necessary to have a detailed description of the singularities by means of suitable a priori estimates. This preliminary analysis is in fact the most difficult part of the procedure.

At the present stage, a good description of the singularities of mean curvature flow is only available for hypersurfaces satisfying certain curvature restrictions. This is in contrast with the Ricci flow, where in low dimensions $(n=2,3)$ it has been possible to study the flow of an arbitrary metric. Our surgery construction applies to a suitable class of hypersurfaces, usually called two-convex. A hypersurface is called $k$-convex, for an integer $k=$ $1, \ldots, n$, if the sum of the smallest $k$ principal curvatures is nonnegative at each point. Also, we need the hypothesis that the surface has dimension $n \geq 3$. Our main result is the following.

TheOREM 1.1. Let $\mathcal{M}_{0} \subset \mathbb{R}^{n+1}$ be a closed immersed $n$-dimensional two-convex hypersurface, with $n \geq 3$. Then there is a mean curvature flow with surgeries with initial value $\mathcal{M}_{0}$ such that, after a finite number of surgeries, the remaining components are diffeomorphic either to $S^{n}$ or to $S^{n-1} \times S^{1}$.

Due to the structure of our surgeries, we can easily deduce that the initial manifold is the connected sum of finitely many components diffeomorphic to $S^{n}$ or to $S^{n-1} \times S^{1}$. Thus we obtain the following classification of 2 -convex hypersurfaces.

Corollary 1.2. Any smooth closed n-dimensional two-convex immersed surface $\mathcal{M} \subset \mathbb{R}^{n+1}$ with $n \geq 3$ is diffeomorphic either to $S^{n}$ or to a finite connected sum of $S^{n-1} \times S^{1}$.

Another consequence of our construction is the following Schoenflies type theorem for simply connected two-convex surfaces.

COROllary 1.3. Any smooth closed simply connected n-dimensional two-convex embedded surface $\mathcal{M} \subset \mathbb{R}^{n+1}$ with $n \geq 3$ is diffeomorphic to $S^{n}$ and bounds a region whose closure is diffeomorphic to a smoothly embedded $(n+1)$-dimensional standard closed ball.

We recall that the Schoenflies conjecture states that any smooth $n$-dimensional embedded surface $\mathcal{M} \subset \mathbb{R}^{n+1}$ diffeomorphic to $S^{n}$ bounds a region whose closure is diffeomorphic to a smoothly embedded $(n+1)$ dimensional standard closed ball. The result has been proved for any dimension except for $n=4$. Thus we show that there are no counterexamples to the conjecture in the class of 2-convex surfaces. The proof of Corollary 1.3 requires some additional argument (like proving that an embedded hypersurface remains embedded under the flow with surgeries) but it is a quite direct consequence of the main Theorem 1.1. 
It is natural to compare the mean curvature flow with surgeries with the generalized solutions mentioned at the beginning. It should be observed that our flow cannot be regarded as a weak solution of the equation, because it introduces an arbitrary modification of the manifold at the surgery time. However, the flow with surgeries is more natural and useful for the geometric and topological applications we are considering. For weak solutions, instead, one does not have such a clear knowledge of the topology of the manifold after the singular time. Let us mention that for the Ricci flow there is no such choice between different approaches, because no notion of weak solution is available there yet.

Let us mention some related results in the previous literature. The structure of $k$-convex hypersurfaces immersed in Riemannian manifolds has been studied by various authors $[\mathbf{1 6}, \mathbf{3 1}, \mathbf{3 7}, \mathbf{4 0}]$. The results of these papers, however, concern mainly the homotopy type of the surfaces. Let us also mention that a piecewise smooth mean curvature flow was considered in [2] for a class of rotationally symmetric hypersurfaces. It was shown there that such surfaces become singular at isolated points and times, splitting into components which become smooth again immediately afterwards, so that no surgery is needed. The analysis depends strongly on the assumption of rotational symmetry. Recently, the construction of a mean curvature flow with surgeries for two-dimensional manifolds, based on independent techniques from [29], has been announced in [9].

In the following we give an informal exposition of the surgery procedure of [29]. The largest part of the paper is devoted to survey the estimates which are necessary for the construction, including the ones of $[\mathbf{2 4 , 2 7 , 2 8}$. We give here not only the statements of the main results, but also an outline of the proofs, where possible. Due to the informal character of the paper, many technical details will be omitted; in particular, most of the proofs should be intended as sketches. We hope that this will suffice to give the reader some understanding of the main techniques used in this theory.

\section{Examples}

There are some well known cases where the solution to the mean curvature flow can be written explicitly, or at least admits a detailed qualitative description. We recall briefly the interesting ones for our purposes. A more detailed description can be found in the monograph by Ecker [11].

EXAMPLE 2.1. Homothetically shrinking solutions These solutions, also called self-similarly shrinking solutions, are those such that $\mathcal{M}_{t}=$ $\lambda(t) \mathcal{M}_{0}$ for a suitable homothety factor $\lambda(t)$ with $\lambda(t)<1$ for $t>0$. The simplest example is the sphere. It is easily checked that, if $\mathcal{M}_{0}=S_{R}^{n}(0)$, the sphere of radius $R$ around the origin, then $\mathcal{M}_{t}=S_{r(t)}^{n}(0)$, where $r(t)=$ $\sqrt{R^{2}-2 n t}$. It follows that the flow exists up to time $T=R^{2} / 2 n$, at which the sphere shrinks to a point. A less obvious example is given by a class of 
immersed curves in the plane, which have been classified by Mullins [33], see also $[\mathbf{1}]$. Other shrinking solutions are obtained by taking products of these surfaces with flat factors, e.g., the cylinder $S^{n-1} \times \mathbb{R}$. It has been proved by Huisken $[\mathbf{2 6}]$ that the ones we have just described are the only homothetically shrinking solutions which are mean convex, i.e., with positive mean curvature everywhere. An interesting example which is not mean convex is the homothetically shrinking torus whose existence was proved by Angenent [5].

EXAMPLE 2.2. Translating graphs A translating graph is a surface $\mathcal{M}_{0}$ which is the graph of some function $y=u(x)$, whose evolution by mean curvature flow exists for all times and is such that $\mathcal{M}_{t}$ is the graph of $y=u(x)+t$. The simplest example is the so called grim reaper, which is the graph of $u(x)=-\ln (\cos x), x \in(-\pi / 2, \pi / 2)$. It is the only example in one dimension, up to translations. In higher dimensions, there is a unique rotationally symmetric example, defined in the whole space and asymptotic to a paraboloid. The existence of translating graphs which are not rotationally symmetric has been recently shown in $[\mathbf{3 8}]$.

EXAmPle 2.3. The standard neckpinch Suppose that $\mathcal{M}_{0}$ looks like two large balls connected by a cylindrical part (neck) which is very thin, in such a way that the mean curvature there is much larger than in the balls. Then one expects that the radius of the neck goes to zero in a short time while the balls move little from their original position. The existence of surfaces with this property was first proved rigorously by Grayson [18]; a simple proof can be found in [11]. An explicit example of initial surface is given in $[\mathbf{2}]$ (see the next example).

In contrast with Example 2.1, the surface here does not become singular everywhere at the singular time, but only in a restricted region. In a case like this it is interesting to define a weak solution after the singular time. One intuitively expects that the surface should divide in two parts, each of them flowing independently afterwards. The idea of the flow with surgeries is to induce this behavior in a controlled way.

Example 2.4. The degenerate neckpinch This example is given in $[\mathbf{2}]$. For a given $\lambda>0$, let us set

$$
\phi_{\lambda}(x)=\sqrt{\left(1-x^{2}\right)\left(x^{2}+\lambda\right)}, \quad-1 \leq x \leq 1 .
$$

For any $n \geq 2$, let $\mathcal{M}^{\lambda}$ be the $n$-dimensional surface in $\mathbb{R}^{n+1}$ obtained by rotation of the graph of $\phi_{\lambda}$. The surface $\mathcal{M}^{\lambda}$ looks like a dumbbell, where the parameter $\lambda$ measures the width of the central part. Then, the following properties hold:

(a) if $\lambda$ is large enough, the surface $\mathcal{M}_{t}^{\lambda}$ eventually becomes convex and shrinks to a point in finite time; 
(b) if $\lambda$ is small enough, $\mathcal{M}_{t}^{\lambda}$ exhibits a neckpinch singularity as in Example 2.3;

(c) there exists $\lambda>0$ such that $\mathcal{M}_{t}^{\lambda}$ shrinks to a point in finite time, has positive mean curvature up to the singular time, but never becomes convex. The maximum of the curvature is attained at the two points where the surface meets the axis of rotation. After rescaling around either of these points, the asymptotic profile of the surface is given by a translating solution of the flow.

The behavior in (c) is called degenerate neckpinch and was first conjectured by Hamilton for the Ricci flow [22, §3]. Intuitively speaking, it is a limiting case of the neckpinch where the cylinder in the middle and the balls on the sides shrink at the same time. One can also build the example in an asymmetric way, with only one of the two balls shrinking simultaneously with the neck, while the other one remains nonsingular. A sharp analysis of the singular behavior for a class of rotationally symmetric surfaces exhibiting a degenerate neckpinch has been done in [6].

Degenerate neckpinches are more difficult to handle when one defines a flow with surgeries, because it is less clear how to find a cylindrical region where the surgery can be performed. Hamilton's intuition for the Ricci flow was that, although the region with the largest curvature is strictly convex, one can find almost cylindrical regions on the surface by moving away by a suitable distance (the rescaled profile near the singularity has an "asymptotically necklike end", in the terminology of $[\mathbf{2 3}]$ ). For the mean curvature flow, one can think of the typical shape of a translating solution, which is asymptotic to a paraboloid. If one considers a strip of the paraboloid far from the vertex, it is close enough to a portion of a cylinder for the purposes of surgery.

\section{Invariance properties}

Let $F: \mathcal{M} \times\left[0, T\left[\rightarrow \mathbb{R}^{n+1}\right.\right.$ be a solution of mean curvature flow (1.1)(1.2) with closed, smoothly immersed evolving surfaces $\mathcal{M}_{t}=F(\cdot, t)(\mathcal{M})$. We denote the induced metric by $g=\left\{g_{i j}\right\}$, the surface measure by $d \mu$, the second fundamental form by $A=\left\{h_{i j}\right\}$ and the Weingarten operator by $W=\left\{h_{j}^{i}\right\}$. We then denote by $\lambda_{1} \leq \cdots \leq \lambda_{n}$ the principal curvatures, i.e., the eigenvalues of $W$, and by $H=\lambda_{1}+\cdots+\lambda_{n}$ the mean curvature. In addition, $|A|^{2}=\lambda_{1}^{2}+\cdots+\lambda_{n}^{2}$ will denote the squared norm of $A$. All these quantities depend on $(p, t) \in \mathcal{M} \times[0, T$ [ and satisfy the following equations computed in $[\mathbf{2 4}]$.

LEMMA 3.1. If $\mathcal{M}_{t}$ evolves by mean curvature flow, the associated quantities introduced above satisfy the following equations (here $\nabla$ and $\Delta$ denote respectively the covariant derivative and the Laplace-Beltrami operator 
induced by the metric on $\left.\mathcal{M}_{t}\right)$ :
(i) $\frac{\partial}{\partial t} g_{i j}=-2 H h_{i j}$
(ii) $\frac{\partial}{\partial t} d \mu=-H^{2} d \mu$
(iii) $\frac{\partial}{\partial t} h_{j}^{i}=\Delta h_{j}^{i}+|A|^{2} h_{j}^{i}$,
(iv) $\frac{\partial}{\partial t} H=\Delta H+|A|^{2} H$,
(v) $\frac{\partial}{\partial t}|A|^{2}=\Delta|A|^{2}-2|\nabla A|^{2}+2|A|^{4}$.

The mean curvature flow is a parabolic system of PDEs and satisfies a local existence and uniqueness result for smooth solutions under general hypotheses. For our purposes the following statement will suffice (see, e.g., $[12,15,17,24])$.

TheOrem 3.2. Let $\mathcal{M}_{0}=F_{0}(\mathcal{M})$ be smooth and closed. Then the mean curvature flow (1.1)-(1.2) has a unique smooth solution, which is defined in a maximal time interval $\left[0, T\left[\right.\right.$, where $0<T<+\infty$, and satisfies $\max _{\mathcal{M}_{t}}|A|^{2} \rightarrow$ $\infty$ as $t \uparrow T$.

A first step in the analysis of singularities is to observe that several geometric properties are invariant under the flow. The invariance can be usually proved in an elementary way by means of the maximum principle. Let us give some examples.

Proposition 3.3. Let $\mathcal{M}_{t}, t \in[0, T)$ be a closed hypersurface evolving by mean curvature flow.

(i) If $H \geq 0$ on $\mathcal{M}_{0}$, then $H>0$ on $\mathcal{M}_{t}$ for any $t \in(0, T)$.

(ii) If $|A|^{2} \leq c H^{2}$ on $\mathcal{M}_{0}$, then $|A|^{2} \leq c H^{2}$ on $\mathcal{M}_{t}$ for any $t \in(0, T)$.

Proof. Part (i) follows from Lemma 3.1 and the strong maximum principle. To obtain (ii), we compute the evolution equation of $f:=|A|^{2} / H^{2}$. We obtain, by Lemma 3.1 and a straightforward computation,

$$
\frac{\partial f}{\partial t}=\Delta f+\frac{2}{H}\langle\nabla H, \nabla f\rangle-\frac{2}{H^{4}}\left|H \nabla_{i} h_{k l}-\nabla_{i} H h_{k l}\right|^{2} .
$$

Thus, the maximum principle implies that the maximum of $f$ is nonincreasing.

Corollary 3.4. Let $\mathcal{M}_{t}, t \in[0, T)$ be a closed $n$-dimensional hypersurface evolving by mean curvature flow.

(i) If $H>0$ on $\mathcal{M}_{0}$, then there is $\varepsilon_{0}>0$ such that $\varepsilon_{0}|A|^{2} \leq H^{2} \leq$ $n|A|^{2}$ everywhere on $\mathcal{M}_{t}$ for all $t \in(0, T)$.

(ii) If $\mathcal{M}_{0}$ has positive scalar curvature, then the same holds for $\mathcal{M}_{t}$ for all $t \in(0, T)$.

Proof. To prove the first inequality in (i), it suffices to take $\varepsilon_{0}=$ $\min _{\mathcal{M}_{0}} H^{2} /|A|^{2}$ which is attained by compactness, and to apply Proposition 3.3-(ii). Inequality $H^{2} \leq n|A|^{2}$ is an algebraic property which holds 
in general. Part (ii) also is a consequence of Proposition 3.3-(ii) because positive scalar curvature is equivalent to $H^{2} /|A|^{2}>1$.

Corollary 3.4-(ii) is a particular case of a more general property of the elementary symmetric polynomials of the curvatures, as we now proceed to show. We recall that the elementary symmetric polynomial of degree $k$ in $n$ variables $\lambda_{1}, \ldots, \lambda_{n}$ is defined as

$$
S_{k}=\sum_{1 \leq i_{1}<i_{2}<\cdots<i_{k} \leq n} \lambda_{i_{1}} \lambda_{i_{2}} \cdots \lambda_{i_{k}}
$$

for $k=1, \ldots, n$. In particular, $S_{1}=H$, and $S_{2}$ is the scalar curvature. It is not difficult to show that

$$
\lambda_{1} \geq 0, \ldots, \lambda_{n} \geq 0 \quad \Longleftrightarrow \quad S_{1} \geq 0, \ldots, S_{n} \geq 0
$$

These polynomials enjoy some remarkable concavity properties, see e.g. [4]. The relevant one for our purposes is the following $[\mathbf{2 8 , 3 0 ]}$.

THEOREM 3.5. Let $\Gamma_{k} \subset \mathbb{R}^{n}$ denote the connected component of $S_{k}>0$ containing the positive cone. Then $S_{l}>0$ on $\Gamma_{k}$ for all $l=1, \ldots, k$ and the quotient $S_{k+1} / S_{k}$ is concave on $\Gamma_{k}$.

The above properties remain unchanged if we regard the polynomials $S_{k}$ as functions of the Weingarten operator, instead of the principal curvatures, because we have the following result, see [3, Lemma 2.2] or [28, Lemma 2.11].

THEOREM 3.6. Let $f\left(\lambda_{1}, \ldots, \lambda_{n}\right)$ be a symmetric convex (concave) function and let $F(A)=f$ (eigenvalues of $A$ ) for any $n \times n$ symmetric matrix $A$ whose eigenvalues belong to the domain of $f$. Then $F$ is convex (concave).

The concavity of the above expressions allows one to apply the maximum principle to obtain invariance properties. This will be clear after deriving the following evolution equation.

Proposition 3.7. Let $F\left(h_{j}^{i}\right)$ be a function homogeneous of degree one. Let $\mathcal{M}_{t}$ be a closed mean convex surface evolving by mean curvature flow such that $h_{j}^{i}$ belongs to the domain of $F$ everywhere. Then

$$
\frac{\partial}{\partial t} \frac{F}{H}-\Delta \frac{F}{H}=\frac{2}{H}\left\langle\nabla H, \nabla \frac{F}{H}\right\rangle-\frac{1}{H} \frac{\partial^{2} F}{\partial h_{j}^{i} \partial h_{l}^{k}} \nabla^{p} h_{i}^{j} \nabla_{p} h_{l}^{k}
$$

As a consequence, if $F$ is concave (convex), any estimate of the form $F \geq c H$ (resp. $F \leq c H)$ is preserved. 
Proof. A straightforward computation, using Lemma 3.1(iii)-(iv) and Euler's theorem on homogeneous functions, yields

$$
\begin{aligned}
\frac{\partial}{\partial t} \frac{F}{H} & =\frac{1}{H} \frac{\partial F}{\partial h_{j}^{i}}\left(\Delta h_{j}^{i}+|A|^{2} h_{j}^{i}\right)-\frac{F}{H^{2}}\left(\Delta H+|A|^{2} H\right) \\
& =\Delta \frac{F}{H}+\frac{2}{H}\left\langle\nabla H, \nabla \frac{F}{H}\right\rangle-\frac{1}{H} \frac{\partial^{2} F}{\partial h_{j}^{i} \partial h_{l}^{k}} \nabla^{p} h_{i}^{j} \nabla_{p} h_{l}^{k} .
\end{aligned}
$$

In particular, the previous proposition can be applied to $F=S_{k+1} / S_{k}$, provided $S_{k} \neq 0$. This leads to the following result, which generalizes Corollary 3.4 .

Proposition 3.8. Let $\mathcal{M}_{0}$ be a closed hypersurface such that $S_{k}>0$ everywhere for a given $k \in\{1, \ldots, n\}$ and let $\mathcal{M}_{t}$ be its evolution by mean curvature flow. Then, for any $l=2, \ldots, k$ there exists $\gamma_{l}$ such that $S_{l} \geq$ $\gamma_{l} H^{l}>0$ on $\mathcal{M}_{t}$ for all $t \in(0, T)$.

Proof. We first observe that on $\mathcal{M}_{0}$ the curvatures $\left(\lambda_{1}, \ldots, \lambda_{n}\right)$ belong everywhere to the set $\Gamma_{k}$ defined in Theorem 3.5. By the same theorem, we have $S_{l}>0$ on $\mathcal{M}_{0}$ for $l=1, \ldots, k$ and so, by compactness, we have $S_{l} \geq c_{l} H S_{l-1}$ for suitable constants $c_{l}>0$, for any $l=2, \ldots, k$. We know from Proposition 3.3 that $H>0$ everywhere on $\mathcal{M}_{t}$ for $t \in(0, T]$. Then we can consider the quotient $S_{2} / H^{2}=S_{2} / S_{1} H$. It is defined for every $t$, it is greater than $c_{2}$ at time zero, and its minimum is nondecreasing by Proposition 3.7. It follows that $S_{2} \geq c_{2} H^{2}$ also for $t \in(0, T)$. We now apply the same procedure to the quotient $S_{3} / S_{2} H$ to conclude that it is greater than $c_{3}$ for $t \in(0, T)$, i.e., $S_{3} \geq c_{3} S_{2} H \geq c_{3} c_{2} H^{3}$. Repeating the argument a finite number of times yields the conclusion.

Further invariance properties for the mean curvature flow can be obtained using Hamilton's maximum principle for tensors [20, Section 4]. Let us first recall a definition. We say that an immersed surface $\mathcal{M}$ is $k$-convex, for some $1 \leq k \leq n$, if the sum of the $k$ smallest curvatures is nonnegative at every point of $\mathcal{M}$. In particular, 1-convexity coincides with convexity, while $n$-convexity means nonnegativity of the mean curvature $H$, i.e., mean convexity. Then we have the following result.

Proposition 3.9. If a closed hypersurface $\mathcal{M}_{0}$ satisfies $\lambda_{1}+\cdots+\lambda_{k} \geq$ $\alpha H$ for some $\alpha \geq 0$ and $1 \leq k \leq n$, then the same holds for its evolution by mean curvature flow $\mathcal{M}_{t}$. In particular, if $\mathcal{M}_{0}$ is $k$-convex, then so is $\mathcal{M}_{t}$.

Proof. The result follows from Hamilton's maximum principle for tensors, provided we show that the inequality $\lambda_{1}+\cdots+\lambda_{k} \geq \alpha H$ describes a convex cone in the set of all matrices, and that this cone is invariant under the system of o.d.e.'s $d h_{j}^{i} / d t=|A|^{2} h_{j}^{i}$, which is obtained by dropping the diffusion term in the evolution equation for the Weingarten operator in Lemma 3.1. 
If we denote by $W\left(v_{1}, v_{2}\right)$ the Weingarten operator applied to two tangent vectors $v_{1}, v_{2}$ at any point, we have

$$
\begin{aligned}
& \lambda_{1}+\cdots+\lambda_{k}=\min \left\{W\left(e_{1}, e_{1}\right)+\cdots+W\left(e_{k}, e_{k}\right):\left\langle e_{i}, e_{j}\right\rangle=\delta_{i j}\right. \\
& \quad \text { for all } 1 \leq i \leq j \leq k\} .
\end{aligned}
$$

This shows that $\lambda_{1}+\cdots+\lambda_{k}$ is a concave function of the Weingarten operator, being the infimum of a family of linear maps. Therefore the inequality $\lambda_{1}+$ $\cdots+\lambda_{k} \geq \alpha H$ describes a convex cone of matrices. In addition, system $d h_{j}^{i} / d t=|A|^{2} h_{j}^{i}$ changes the Weingarten operator by homotheties, and thus leaves any cone invariant. The conclusion follows.

In particular, we obtain that convex surfaces remain convex under the flow. Observe that the same property also follows from Proposition 3.8 by taking $k=n$ and keeping into account property (3.2).

\section{Convergence to a point of convex surfaces}

As we recalled in the introduction, the singular behavior of convex surfaces under the flow is described by the following result.

THEOREM 4.1. Let $\mathcal{M}_{0}$ be an $n$-dimensional closed convex surface embedded in $\mathbb{R}^{n+1}$. Then $\mathcal{M}_{t}$ shrinks to a point as $t \rightarrow T$. In addition, if we choose a suitable rescaling factor $\rho(t)$, then the surfaces $\rho(t) \mathcal{M}_{t}$ converge to a sphere as $t \rightarrow T$.

Proof The above theorem was proved by Huisken in $[24]$ in the case $n \geq 2$ and by Gage and Hamilton [17] when $n=1$. Although the result in $[24]$ is well known, it is worth describing here some of the main ideas in the proof, since they play an important role in the later developments of the theory. Let us set

Then it is easy to check that

$$
f=\frac{|A|^{2}}{H^{2}}-\frac{1}{n}
$$

$$
f H^{2}=\sum_{i<j}\left(\lambda_{i}-\lambda_{j}\right)^{2} .
$$

Thus, $f$ is nonnegative and it measures how much the curvatures differ from each other. It vanishes identically on a surface if and only if the surface is a sphere. This approach is suggested by [19, §8], where Hamilton considered a similar function of the eigenvalues of the Ricci tensor.

We have seen in Proposition 3.3 that the maximum of $f$ is nonincreasing. To prove convergence to a sphere one needs some stronger estimate, showing that $f$ tends to zero as the singular time is approached. Following [19], one considers the function $f_{\sigma}=f H^{\sigma}$ for a suitably small $\sigma>0$. Observe that $f_{\sigma}$ is a homogeneous function of the curvatures of degree $\sigma>0$; thus, one 
would expect $f_{\sigma}$ to blow up as the singular time $T$ is approached. The next theorem shows instead that it remains bounded, and this is one of the crucial steps in the proof of Theorem 4.1.

THEOREM 4.2. If $\sigma>0$ is small enough, the function $f_{\sigma}$ is uniformly bounded for $t \in[0, T)$.

Proof. Let us first remark that a similar result holds for the analogous function considered in [19] for the Ricci flow. However, the method of proof is quite different. In fact, the result of [19] follows from an application of the maximum principle. In our case, instead, the additional factor $H^{\sigma}$ induces the presence of a positive zero order term in the evolution equation for $f_{\sigma}$ that cannot be directly compensated by the other terms. More precisely, one finds

$$
\frac{\partial f_{\sigma}}{\partial t} \leq \Delta f_{\sigma}+\frac{2(1-\sigma)}{H}\left\langle\nabla H, \nabla f_{\sigma}\right\rangle-\frac{2}{H^{4-\sigma}}\left|H \nabla_{i} h_{k l}-h_{k l} \nabla_{i} H\right|+\sigma|A|^{2} f_{\sigma} .
$$

Thus, a more elaborate procedure is needed to estimate $f_{\sigma}$. Let us first state a useful lower bound for the gradient term in the above inequality. One can prove that on convex surfaces (and in fact under more general hypotheses) there exists $c$ such that

$$
\left|H \nabla_{i} h_{k l}-h_{k l} \nabla_{i} H\right| \geq c H^{2}|\nabla H|^{2}
$$

(see [24, Lemma 2.3]). We now integrate the inequality on the manifold and try to estimate the $L^{p}$ norm of $f_{\sigma}$. After integrating by parts we obtain

$$
\begin{aligned}
\frac{d}{d t} \int_{\mathcal{M}_{t}} f_{\sigma}^{p} d \mu \leq & -\frac{p(p-1)}{2} \int_{\mathcal{M}_{t}} f_{\sigma}^{p-2}\left|\nabla f_{\sigma}\right|^{2} d \mu-\frac{p}{c} \int_{\mathcal{M}_{t}} \frac{f_{\sigma}^{p-1}}{H^{2-\sigma}}|\nabla H|^{2} d \mu \\
& +p \sigma \int_{\mathcal{M}_{t}}|A|^{2} f_{\sigma}^{p} d \mu .
\end{aligned}
$$

To show that the last term can be compensated by the other two, we need some estimate involving both zero order curvature terms and gradient terms. To this purpose, we recall the identity [24, Lemma 2.1]

$$
\frac{1}{2} \Delta|A|^{2}=\left\langle h_{i j}, \nabla_{i} \nabla_{j} H\right\rangle+|\nabla A|^{2}+Z,
$$

where $Z=H \sum \lambda_{i}^{3}-\left(\sum \lambda_{i}^{2}\right)^{2}$. Using this equality, one can compute

$$
\begin{aligned}
\Delta f_{\sigma} \geq & \frac{2}{H^{2-\sigma}} h^{i j} \nabla_{i} \nabla_{j} H+\frac{2}{H^{2-\sigma}} Z \\
& -\frac{2(1-\sigma)}{H}\left\langle\nabla H, \nabla f_{\sigma}\right\rangle+\left(\frac{2}{n H^{1-\sigma}}-\frac{2-\sigma}{H} f_{\sigma}\right) \Delta H .
\end{aligned}
$$


After integrating this inequality on $\mathcal{M}_{t}$ and performing some standard computations we obtain that, for all $\eta>0$

$$
\begin{aligned}
\int \frac{1}{H^{2-\sigma}} f_{\sigma}^{p-1} Z d \mu \leq & (2 \eta p+5) \int \frac{1}{H^{2-\sigma}} f_{\sigma}^{p-1}|\nabla H|^{2} d \mu \\
& +\eta^{-1}(p-1) \int f_{\sigma}^{p-2}\left|\nabla f_{\sigma}\right|^{2} d \mu
\end{aligned}
$$

On the other hand, it can be shown $[\mathbf{2 4}$, Lemma 2.3(i)] that on a uniformly convex surface, say $h_{i j} \geq \varepsilon H g_{i j}$, we have

$$
Z \geq n \varepsilon^{2} H^{4-\sigma} f_{\sigma}>n \varepsilon^{2}|A|^{2} H^{2-\sigma} f_{\sigma} .
$$

Thus we can combine estimates (4.3) and (4.5) with an appropriate choice of $\eta$ to show that, for $p$ suitably large and for $\sigma$ suitably small the $L^{p}$ norm of $f_{\sigma}$ is decreasing in time.

This property is the starting point for a Stampacchia iteration procedure to obtain that the $L^{\infty}$ norm of $f_{\sigma}$ is bounded. The proof also relies on the Michael-Simon Sobolev inequality [32]. For the details, see $[\mathbf{2 4}, \S 5]$.

Several steps remain to complete the proof of Theorem 4.1. Roughly speaking, the above result shows that, at the points where the curvature becomes unbounded, the Weingarten operator approaches the one of a sphere. One then needs to show that the curvature becomes unbounded in the whole surface when the singular time is approached. The main steps are a gradient estimate for the mean curvature and an application of Myers' theorem, see $[24]$.

\section{Convexity estimates for mean convex surfaces}

We shall now consider the formation of singularities for surfaces which are mean convex, that is, with positive mean curvature everywhere. As we have seen in Theorem 3.3, this property is preserved by the mean curvature flow. For the study of singularities, mean convexity is a significant generalization of convexity. For instance, it is enough general to allow for the neckpinch behavior described in Section 2; in particular, mean convex surfaces do not necessarily shrink to a point at the singular time. A fundamental result in the analysis of singularities of mean convex surfaces is the following estimate on the elementary symmetric polynomials of the curvatures, proved in $[\mathbf{2 8}]$.

ThEOREM 5.1. Let $\mathcal{M}_{0} \subset \mathbb{R}^{n+1}$ be a closed mean convex immersed hypersurface and let $\mathcal{M}_{t}, t \in[0, T)$ be its evolution by mean curvature flow. Then, for any $\eta>0$ there exists $C=C\left(\eta, \mathcal{M}_{0}\right)$ such that $S_{k} \geq-\eta H^{k}-C$ for any $k=2, \ldots, n$ on $\mathcal{M}_{t}$ for any $t \in[0, T)$.

Such an estimate easily implies the following one, which has a more immediate interpretation. 
THEOREM 5.2. Under the same hypotheses of the previous theorem, for any $\eta>0$ there exists $C=C\left(\eta, \mathcal{M}_{0}\right)$ such that $\lambda_{1} \geq-\eta H-C$ on $\mathcal{M}_{t}$ for any $t \in[0, T)$.

The interest of the above estimate lies in the fact that $\eta$ can be chosen arbitrarily small and $C$ is a constant not depending on the curvatures. Thus we see that, roughly speaking, the negative curvatures become negligible with respect to the others when the singular time is approached. This implies that the surface becomes asymptotically convex near a singularity. For this reason we call the estimates of the theorems above convexity estimates. Let us observe that the result of Theorem 5.2 is very similar to a well-known estimate in the Ricci flow, usually called Hamilton-Ivey estimate [22, Theorem 24.4]. In contrast to our result, Hamilton-Ivey estimate holds for arbitrary manifolds, but only in the three dimensional case. Observe that Theorem 5.2 cannot be valid for general surfaces even in low dimensions, because the property is violated in Angenent's example of self-similar shrinking torus.

In this section we illustrate the main steps in the proof of Theorem 5.1. For a better understanding of the technique, it is useful to consider first the following weaker result.

THEOREM 5.3. Let $\mathcal{M}_{0}$ be a closed hypersurface such that, for some $1 \leq k \leq n-1$, we have $S_{1}>0, \ldots, S_{k}>0$ everywhere on $\mathcal{M}_{0}$. Then, for any $\eta>0$ there exists $C=C\left(\eta, \mathcal{M}_{0}\right)$ such that $S_{k+1} \geq-\eta H^{k+1}-C$ everywhere on $\mathcal{M}_{t}$ for any $t \in[0, T)$.

Proof. The case $k=1$ of this theorem (which can be treated in a more explicit way) was proved in [27]. The general case can be regarded as a simplified version of the main theorem in [28]. The strategy of proof is similar to the one of Theorem 4.2. By our assumptions and by Proposition 3.8, we have $S_{k}>c_{k} H^{k}$ everywhere on $\mathcal{M}_{t}$ and so the quotient $Q_{k+1}:=S_{k+1} / S_{k}$ is well defined. Let us consider the function

$$
f=f_{\sigma, \eta}=\frac{-Q_{k+1}-\eta H}{H^{1-\sigma}} .
$$

where $\sigma, \eta>0$. A straightforward calculation yields the evolution equation

$$
\begin{aligned}
\frac{\partial f}{\partial t}= & \Delta f+\frac{2(1-\sigma)}{H}\langle\nabla H, \nabla f\rangle-\frac{\sigma(1-\sigma)}{H^{2}} f|\nabla H|^{2} \\
& +\frac{1}{H^{1-\sigma}} \frac{\partial^{2} Q_{k+1}}{\partial h_{j}^{i} \partial h_{q}^{p}} \nabla^{m} h_{j}^{i} \nabla_{m} h_{q}^{p}+\sigma|A|^{2} f .
\end{aligned}
$$

The function $f_{\eta, \sigma}$ will play a similar role to $f_{\sigma}$ in the proof of Theorem 4.1. Actually, in the case $k=1$ the two functions essentially coincide. Even in this case, however, the proof needs to be modified, because we no longer have the convexity assumption that was crucial in some of the estimates there.

In the case $\sigma=0$, the maximum principle applied to (5.1) and the concavity of $Q_{k+1}$ give a bound on $f_{\sigma, \eta}$. However, the interesting case for us is 
when $\sigma>0$. Namely, we need to show that for any $\eta>0$ there exists $\sigma>0$ such that $f_{\sigma, \eta}$ is bounded. In fact, proving that $f_{\sigma, \eta}<C$ for some $C>0$ implies that

$$
S_{k+1} \geq S_{k}\left(-\eta H-C H^{1-\sigma}\right) \geq-2 \eta H^{k+1}-C^{\prime}
$$

for a suitable $C^{\prime}=C^{\prime}(C, \sigma, \eta)$. Since $\eta>0$ is arbitrary, this proves Theorem 5.3.

To prove the boundedness of $f$ we are going to apply the same technique of Theorem 4.2, consisting of $L^{p}$ estimates on $f$ followed by an iteration technique. Note that, in contrast to that theorem, the function $f$ here is not positive everywhere: it is negative, for instance, at all convex points of the surface. It will be convenient to prove estimates on $f_{+}^{p}$, where $f_{+}$is the positive part of $f$. In this way, we only have to consider the points of $\mathcal{M}_{t}$ where $f>0$, that is, where $Q_{k+1} \leq-\eta H$.

As a first step, we need to show that the gradient term in the second line of (5.1) is not only non positive, but it has some coercivity, i.e. we need, an analogue of inequality (4.2). This is not an obvious property, because the function $Q_{k+1}$ is homogeneous of degree one, and thus it is not strictly concave. However, analyzing carefully the properties of $Q_{k+1}$ and exploiting the symmetries given by the Codazzi equations we obtain $[\mathbf{2 8}$, Theorems 2.5 and 2.14] that for any $\eta>0$ there exists $c>0$ such that

$$
\frac{\partial^{2} Q_{k+1}}{\partial h_{j}^{i} \partial h_{q}^{p}} \nabla^{m} h_{j}^{i} \nabla_{m} h_{q}^{p} \leq-c \frac{|\nabla A|^{2}}{|A|}
$$

at all points where $Q_{k+1}<-\eta H$. This allows to obtain from (5.1)

$$
\begin{aligned}
\frac{d}{d t} \int_{\mathcal{M}_{t}} f_{+}^{p} d \mu \leq & -\frac{1}{2} p(p-1) \int_{\mathcal{M}_{t}} f_{+}^{p-2}|\nabla f|^{2} d \mu-C p \int_{\mathcal{M}_{t}} \frac{f_{+}^{p-1}}{H^{2-\sigma}}|\nabla A|^{2} \\
& +2 p \sigma \int_{\mathcal{M}_{t}} H^{2} f_{+}^{p} d \mu
\end{aligned}
$$

for a suitable $C=C(\eta)$. In the above arguments it is important that we can restrict our attention to the points where $Q_{k+1}<-\eta H$. This allows us to avoid the points where $Q_{k+1}=0$ and the function $Q_{k+1}$ has weaker concavity properties. Observe, for instance, that $S_{k+1}=0$ at all $\left(\lambda_{1}, \ldots, \lambda_{n}\right)$ with $\lambda_{1}=\lambda_{2}=\cdots=\lambda_{n-k}=0$ and the other entries are arbitrary. This shows that the hessian of $Q_{k+1}$ has a large kernel at such points.

Now we need an inequality which allows to estimate zero order terms by first order terms, analogue to (4.5). To this purpose, we look for a suitable identity involving derivatives of the curvature together with zero order curvature terms. It turns out that it is convenient to consider the quantity

$$
\frac{\partial S_{k}}{\partial h_{i j}} \nabla_{i} \nabla_{j} S_{k+1} .
$$


A long but elementary computation, which uses the commutator identities for covariant derivatives and various properties of the elementary symmetric polynomials [28, Lemma 2.15], yields the identity

$$
\begin{aligned}
\frac{\partial S_{k}}{\partial h_{i j}} \nabla_{i} \nabla_{j} S_{k+1}= & \frac{\partial S_{k}}{\partial h_{i j}} \frac{\partial^{2} S_{k+1}}{\partial h_{l m} \partial h_{p q}} \nabla_{i} h_{l m} \nabla_{j} h_{p q}+\frac{\partial S_{k}}{\partial h_{i j}} \frac{\partial S_{k+1}}{\partial h_{l m}} \nabla_{l} \nabla_{m} h_{i j} \\
& -H S_{k} S_{k+1}+(k+1) S_{k+1}^{2}+k\left[(k+1) S_{k+1}^{2}\right. \\
& \left.-(k+2) S_{k} S_{k+2}\right] .
\end{aligned}
$$

Now we have $(k+1) S_{k+1}^{2}-(k+2) S_{k} S_{k+2} \geq 0$ by a classical property of symmetric polynomials called Newton's inequality. We deduce that, at all points where $Q_{k+1}<-\eta H$, we have

$$
\begin{aligned}
\eta H^{2} S_{k}^{2}< & -H S_{k}^{2} Q_{k+1}=-H S_{k} S_{k+1} \\
< & \frac{\partial S_{k}}{\partial h_{i j}} \nabla_{i} \nabla_{j} S_{k+1}-\frac{\partial S_{k}}{\partial h_{i j}} \frac{\partial^{2} S_{k+1}}{\partial h_{l m} \partial h_{p q}} \nabla_{i} h_{l m} \nabla_{j} h_{p q} \\
& -\frac{\partial S_{k}}{\partial h_{i j}} \frac{\partial S_{k+1}}{\partial h_{l m}} \nabla_{l} \nabla_{m} h_{i j} .
\end{aligned}
$$

Let us simply denote by $(R H S)$ the right hand side of this inequality. We have

$$
\int_{\mathcal{M}_{t}} H^{2} f_{+}^{p} d \mu \leq c_{k}^{-2} \int_{\mathcal{M}_{t}} H^{2} S_{k}^{2} H^{-2 k} f_{+}^{p} d \mu \leq c_{k}^{-2} \eta^{-1} \int_{\mathcal{M}_{t}}(R H S) \cdot H^{-2 k} f_{+}^{p} d \mu .
$$

where the last integral no longer contains zero order curvature terms. A careful computation involving integration by parts and properties of the polynomial $S_{k}[\mathbf{2 8}$, Proposition 3.6] then shows that the negative gradient terms in (5.2) can compensate the positive term for a suitable choice of the constants. In this way we prove that, for any $\eta>0$ and $p$ large enough, there exists $\sigma>$ 0 such that the $L^{p}$ norm of $\left(f_{\sigma, \eta}\right)_{+}$is nonincreasing. This allows to apply the same iteration procedure as in the proof of Theorem 4.2 to conclude that $f_{\sigma, \eta}$ is bounded from above for a suitable $\sigma>0$, and this proves the theorem.

The above statement contains the strong assumption of the positivity of $S_{l}$ for $l=1, \ldots, k$. To generalize the technique to the case where we only have $H>0$, we define a suitable perturbation of the second fundamental form. For given $\varepsilon, D>0$, we define $b_{i j}=b_{i j ; \varepsilon, D}$ as follows

$$
b_{i j}=h_{i j}+(\varepsilon H+D) g_{i j} .
$$

We denote by $\tilde{S}_{k}$ the symmetric polynomials computed with respect to $b_{i j}$ instead of $h_{i j}$ (we do not write explicitly the dependence on $\varepsilon, D$ for simplicity of notation). The interest of this definition is shown by the next result [28, Lemmas 2.8, 2.11]. 
Proposition 5.4. Given a mean convex hypersurface and $k \in\{2, \ldots, n\}$, the following properties hold.

(i) Suppose that for any $\eta>0$ there exists $C$ such that $S_{l}=-\eta H^{l}-C$, for $l=2, \ldots, k$. Then, for any $\varepsilon>0$ there exists $D$ such that $\tilde{S}_{l}>0$ for $l=2, \ldots, k$.

(ii) Conversely, suppose that for any $\varepsilon>0, \eta>0$ there exist $D, C$ such that $\tilde{S}_{l}>-\eta \tilde{H}-C$ for $l=2, \ldots, k$. Then, for any $\eta>0$ there exists $K$ (in general larger than $C$ ) such that $S_{l}=-\eta H^{l}-K$, for $l=2, \ldots, k$.

Proof of Theorem 5.1. The perturbation described above allows us to apply an induction procedure similar to the one of Proposition 3.8. We first apply Theorem 5.3 for $k=1$ and obtain that for any $\eta>0$ there exists $C>0$ such that $S_{2} \geq-\eta H^{2}-C$. By Proposition 5.4, the perturbed polynomial $\tilde{S}_{2}$ is positive, and so we can consider the quotient $\tilde{S}_{3} / \tilde{S}_{2}$. Suppose that the proof of Theorem 5.3 can be carried through also for the perturbed polynomials. Then we obtain that for any $\eta>0$ there exists $C$ such that $\tilde{S}_{3} \geq-\eta \tilde{H}^{3}-C$. But then the unperturbed polynomial $S_{3}$ satisfies the same estimate, although with a larger constant, by part (ii) of Proposition 5.4. This shows that the procedure can be iterated to show that all polynomials $S_{k}$ up to $k=n$ satisfy the desired estimate.

The difficult part is to check that the proof of Theorem 5.3 indeed applies also to the perturbed polynomials. The perturbation induces the presence of several additional terms in the equation, some of which require a sharp estimation. The computations become more involved, but it turns out that the same procedure works. Roughly speaking, the additional terms due to $\varepsilon$ can be made arbitrarily small by choosing $\varepsilon$ close enough to zero, while the ones containing $D$ are negligible because they are of lower order.

As it is customary in many nonlinear PDEs, it is possible to study the singular behavior of surfaces evolving by mean curvature flow by rescaling techniques. The property of rescalings are described in the references $[\mathbf{2 6}$, $\mathbf{2 7}, \mathbf{2 8}$ ] and are not strictly needed for the results described in the remainder of the paper. However, we recall them briefly here since they are useful to have a better insight of the surgery procedure.

In the rescaling procedure one dilates in space and time the flow around the points of a sequence along which the curvature becomes unbounded. The dilations are such that the rescaled flows satisfy local uniform curvature bounds and so we have convergence of a subsequence to a smooth limiting mean curvature flow. A precise description of the procedure is given in $[\mathbf{2 7}]$, see also $[\mathbf{2 2}, \S 16]$. In the rescaling procedure the constant term in the convexity estimates of Theorem 5.1 disappears and we obtain that any limiting flow satisfies $\lambda_{1} \geq-\eta H$ for arbitrary $\eta>0$, that is, $\lambda_{1} \geq 0$. 
Thus we have

Corollary 5.5. Any limit obtained by rescaling near a singularity a mean convex surface evolving by mean curvature flow is convex (not necessarily strictly).

The above corollary has been also obtained by White [39] by completely different techniques. His approach also applies to weak solutions. The following result gives a classification of the possible limits obtained by rescaling near a singularity in the mean convex case.

TheOREM 5.6. Let $\mathcal{M}_{t} \subset \mathbb{R}^{n+1}$ be a mean curvature flow of closed mean convex hypersurfaces. Then there is a sequence of rescaled flows near the singular time converging to one of the following flows:

(i) a product of the form $S_{t}^{n-k} \times \mathbb{R}^{k}$, for some $0 \leq k \leq n-1$ where $S_{t}^{n-k}$ is an $(n-k)$-dimensional shrinking sphere;

(ii) a flow of the form $G_{t} \times \mathbb{R}^{n-1}$, where $G_{t}$ is a homothetically shrinking curve in the plane;

(iii) a flow of the form $\Gamma_{t}^{n-k} \times \mathbb{R}^{k}$, for some $0 \leq k \leq n-1$, where $\Gamma_{t}^{n-k}$ is an $(n-k)$-dimensional strictly convex translating solution to the flow.

The above result is proved in [26, Theorem 5.1] and [28, Theorem 4.1]. In addition to the convexity estimates, this classification relies on two other important results, namely Huisken's monotonicity formula [25] and Hamilton's differential Harnack inequality [21].

\section{Cylindrical and gradient estimates for two-convex surfaces}

From now on we consider mean curvature flow of hypersurfaces which have dimension $n \geq 3$ and are uniformly 2-convex, that is, satisfy $\lambda_{1}+\lambda_{2} \geq \alpha H$ everywhere for some $\alpha>0$. As we have seen in Proposition 3.9 , this property is preserved by the flow. The motivation for considering 2-convex surfaces can be intuitively understood in view of the classification of the possible profiles in Theorem 5.6. If our evolving surfaces are uniformly 2 -convex, then so is any limit of rescaled flows. This restricts the number of possibilities in Theorem 5.6, since the only uniformly two-convex limits are the sphere $S^{n}$, the cylinder $S^{n-1} \times \mathbb{R}$ and the $n$-dimensional translating solutions $\Gamma_{t}^{n}$. All these profiles are compatible with the surgery procedure we are willing to define. In fact, if the limit of the rescalings is the sphere $S^{n}$, then the original manifold should be diffeomorphic to a sphere, and no surgery is needed since the topology is known. If the limit is a cylinder, then the manifold should possess a cylindrical region where we can do surgery. If it is an $n$-dimensional translating solution $\Gamma_{t}^{n}$, we also expect to find a cylindrical region, as explained at the end of $\S 2$. This discussion should be regarded only as a heuristic motivation, since the information provided by 
Theorem 5.6 is too weak for the purposes of a flow with surgeries. Our actual proof will be independent of Theorem 5.6 and in fact will not use rescaling techniques, except at one stage (Theorem 9.1). We begin with the following result.

TheOREM 6.1. Let $\mathcal{M}_{t}$, with $t \in[0, T)$, be a closed 2 -convex solution of mean curvature flow. Then, for any $\eta>0$ there exists a constant $C_{\eta}$ such that

$$
\left|\lambda_{1}\right| \leq \eta H \Longrightarrow\left|\lambda_{j}-\lambda_{k}\right| \leq c \eta H+C_{\eta}, \quad j, k>1
$$

everywhere on $\mathcal{M}_{t}$, for $t \in[0, T)$, where $c$ only depends on $n$.

We call the above result a cylindrical estimate because it shows that, at a point where $H$ is large and $\lambda_{1} / H$ is small, the Weingarten operator is close to the one of a cylinder, since it has all eigenvalues close to each other except for $\lambda_{1}$ which is small. Such a property is an important tool for the detection of the cylindrical regions where the surgeries will be performed.

To derive this estimate, we consider again the quotient $|A|^{2} / H^{2}$ which was used in the proof of Theorem 4.1. On a cylinder $\mathbb{R} \times S^{n-1}$ we have $|A|^{2} / H^{2} \equiv 1 /(n-1)$. The converse does not hold, that is, if we have $|A|^{2} / H^{2}=1 /(n-1)$ at one point, this does not imply that the curvatures are a multiple of the ones of a cylinder. However, if $|A|^{2} / H^{2}=1 /(n-1)$ and in addition $\lambda_{1}=0$, then necessarily $\lambda_{2}=\cdots=\lambda_{n}$. In fact, we have the identity

$$
|A|^{2}-\frac{1}{n-1} H^{2}=\frac{1}{n-1}\left(\sum_{1<i<j}\left(\lambda_{i}-\lambda_{j}\right)^{2}+\lambda_{1}\left(n \lambda_{1}-2 H\right)\right) .
$$

In view of this equality, the estimate of Theorem 6.1 is an immediate consequence of the next result $[\mathbf{2 9}]$.

TheOREM 6.2. Let $\mathcal{M}_{t}, t \in[0, T)$, be a closed 2 -convex solution of mean curvature flow. Then, for any $\eta>0$ there exists a constant $C_{\eta}>0$ such that

$$
|A|^{2}-\frac{H^{2}}{n-1} \leq \eta H^{2}+C_{\eta}
$$

on $\mathcal{M}_{t}$ for any $t \in[0, T)$.

Proof. Let us consider, for $\eta \in \mathbb{R}$ and $\sigma \in[0,2]$, the function

$$
f_{\sigma, \eta}=\frac{|A|^{2}-\left(\frac{1}{n-1}+\eta\right) H^{2}}{H^{2-\sigma}} .
$$

Such a function is very similar to $f_{\sigma}$ considered in the proof of Theorem 4.1, and in fact it satisfies the same inequality (4.1). However, in this case we 
do not have a bound from below for $Z$ analogous to (4.6). In fact, $Z$ can be negative on nonconvex surfaces. A typical example is when $\lambda_{1}<0$ and $\lambda_{2}=\ldots=\lambda_{n}>0$; then $Z<0$, even if $\left|\lambda_{1}\right|$ is small compared to the other curvatures.

However, using also the convexity estimate of Theorem 5.2, we can show [29, Lemma 5.2] that there exists a constant $\gamma_{1}>0$ with the following property: for any $\delta>0$ there exists $K_{\delta}$ such that

$$
Z \geq \gamma_{1} H^{2}\left(|A|^{2}-\frac{1}{n-1} H^{2}-\delta H^{2}\right)-K_{\delta} H^{3}
$$

on $\mathcal{M}_{t}$ for any $t>0$. As in the proof of Theorem 5.3, we will estimate the $L^{p}$ norms of the positive part $\left(f_{\sigma, \eta}\right)_{+}$. In this way, we only need to consider the points where the positive part is nonzero, i.e., $|A|^{2}-\frac{H^{2}}{n-1} \geq \eta H^{2}$. Thus, if we choose $\delta=\eta / 2$ in (6.3) the first term is positive and the only negative contribution to the right hand side is the last term which has lower order. It turns out that this is enough to apply the usual iteration technique of the previous theorems and obtain an upper bound for $f_{\sigma, \eta}$, see $[\mathbf{2 9}$, Theorem 5.3]. Such a bound easily implies the estimate of Theorem 6.2.

Observe that similar results have been obtained by Hamilton for the Ricci flow of arbitrary three-dimensional manifolds in [22, Theorem 24.7] and of four-dimensional manifolds with positive isotropic curvature in [23, Theorem B3.3].

We next describe an estimate for the gradient of the curvature for our evolving surfaces. With respect to the gradient estimates for mean curvature flow already available in the literature, e.g., $[\mathbf{1 0}, \mathbf{1 2}]$, the estimate here does not depend on the maximum of the curvature in some neighborhood of the point under consideration. To prove this result we need to assume that the surfaces are 2-convex and that their dimension is at least three.

TheOREm 6.3. Let $\mathcal{M}_{t}, t \in[0, T)$, be a closed $n$-dimensional 2 -convex solution of mean curvature flow, with dimension $n \geq 3$. Then there is a constant $\gamma_{2}=\gamma_{2}(n)$ and a constant $\gamma_{3}=\gamma_{3}\left(n, \mathcal{M}_{0}\right)$ such that the flow satisfies the uniform estimate

$$
|\nabla A|^{2} \leq \gamma_{2}|A|^{4}+\gamma_{3}
$$

for every $t \in[0, T)$.

Proof. The result is obtained by applying the maximum principle to a suitable function we are going to introduce. An important tool in the proof is the inequality [24, Lemma 2.1], valid for any immersed hypersurface,

$$
|\nabla A|^{2} \geq \frac{3}{n+2}|\nabla H|^{2} .
$$


Observe that $\frac{3}{n+2}>\frac{1}{n-1}$ if $n \geq 3$. Let us set

$$
\kappa_{n}=\frac{1}{2}\left(\frac{3}{n+2}-\frac{1}{n-1}\right) .
$$

By Theorem 6.2 there exists $C_{0}>0$ such that

$$
\left(\frac{1}{n-1}+\kappa_{n}\right) H^{2}-|A|^{2}+C_{0} \geq 0
$$

Let us set

$$
g_{1}=\left(\frac{1}{n-1}+\kappa_{n}\right) H^{2}-|A|^{2}+2 C_{0}, \quad g_{2}=\frac{3}{n+2} H^{2}-|A|^{2}+2 C_{0} .
$$

Then we have $g_{2}>g_{1} \geq C_{0}$, and so $g_{i}-2 C_{0}=2\left(g_{i}-C_{0}\right)-g_{i} \geq-g_{i}$ for $i=1,2$. Using the evolution equations for $|A|^{2}, H^{2}$ (see Lemma 3.1) and inequality (6.5) we find

$$
\begin{aligned}
\frac{\partial}{\partial t} g_{1}-\Delta g_{1} & =-2\left(\left(\frac{1}{n-1}+\kappa_{n}\right)|\nabla H|^{2}-|\nabla A|^{2}\right)+2|A|^{2}\left(g_{1}-2 C_{0}\right) \\
& \geq 2\left(1-\frac{n+2}{3}\left(\frac{1}{n-1}+\kappa_{n}\right)\right)|\nabla A|^{2}-2|A|^{2} g_{1} \\
& =2 \kappa_{n} \frac{n+2}{3}|\nabla A|^{2}-2|A|^{2} g_{1} .
\end{aligned}
$$

Similarly

$$
\frac{\partial}{\partial t} g_{2}-\Delta g_{2}=-2\left(\frac{3}{n+2}|\nabla H|^{2}-|\nabla A|^{2}\right)+2|A|^{2}\left(g_{2}-2 C_{0}\right) \geq-2|A|^{2} g_{2} \text {. }
$$

In addition (see $[\mathbf{2 4}$, Theorem 7.1])

$$
\frac{\partial}{\partial t}|\nabla A|^{2}-\Delta|\nabla A|^{2} \leq-2\left|\nabla^{2} A\right|^{2}+c_{n}|A|^{2}|\nabla A|^{2},
$$

for a constant $c_{n}$ depending only on $n$. Using the above relations one obtains, after a straightforward computation, the following inequality for the quotient $|\nabla A|^{2} / g_{1} g_{2}$ :

$$
\begin{gathered}
\frac{\partial}{\partial t}\left(\frac{|\nabla A|^{2}}{g_{1} g_{2}}\right)-\Delta\left(\frac{|\nabla A|^{2}}{g_{1} g_{2}}\right)-\frac{2}{g_{2}}\left\langle\nabla g_{2}, \frac{|\nabla A|^{2}}{g_{1} g_{2}}\right\rangle \\
\leq \frac{|\nabla A|^{2}|A|^{2}}{g_{1} g_{2}}\left(\left(c_{n}+4\right)-2 \kappa_{n}^{2} \frac{n+2}{3 n} \frac{|\nabla A|^{2}}{g_{1} g_{2}}\right) .
\end{gathered}
$$

The maximum principle then implies that $|\nabla A|^{2} \leq c_{1} g_{1} g_{2}$, where $c_{1}$ only depends on $n$ and on the initial data. Using the definition of $g_{1}, g_{2}$, this easily yields our assertion. 
Once the estimate for $|\nabla A|$ is obtained, it is easy to obtain similar estimates for the higher order derivatives, as well as the time derivatives. In particular, we have

$$
\left|\partial_{t} A\right| \leq c_{1}|A|^{3}+c_{2}
$$

Let us note that at this stage no analogous direct a priori estimates for the derivatives of the curvature are known for Ricci flow, since the corresponding estimates obtained by Perelman $[\mathbf{3 4}, \mathbf{3 5}]$ are derived via contradiction arguments.

\section{The surgery procedure}

We now describe in more detail how we are going to perform our surgeries. As explained in the introduction, the surgery consists of removing an almost cylindrical region (called a neck) and replacing it by two convex caps. It is important that the procedure does not alter the validity of the estimates proved in the previous sections. To check this, it is necessary to

- give a precise definition of neck, specifying the notion of "being close to a cylinder" in a quantitative way;

- give an explicit expression of the surface after the surgery;

- show that the estimates of the previous sections remain valid after the surgeries with the same constants.

These steps are carefully carried out by Hamilton [23] in the case of the Ricci flow. We have followed his approach in many parts, with some modifications suggested by our framework of immersed surfaces. Since the complete definitions are lengthy, we give a simplified exposition by omitting most of the technical details.

Hamilton [23] gives different notions of necks in the case where $\mathcal{M}$ is an abstract Riemannian manifold. For a given $\varepsilon>0$, he defines $\varepsilon$-geometric necks as diffeomorphism $N: S^{n-1} \times[a, b] \rightarrow \mathcal{M}$ such that the standard metric on the cylinder and the pull-back of the metric on $\mathcal{M}$ to the cylinder are $\varepsilon$-close, up to a homothety. By " $\varepsilon$-close" we mean that the norm of the difference of the two metric tensors (measured with respect to the standard metric of the cylinder) is everywhere less than $\varepsilon$. He then defines $\varepsilon$-curvature necks as regions of $\mathcal{M}$ where the curvature operator is $\varepsilon$-close at every point, up to a homothety, to the curvature operator of a standard cylinder. The first notion is useful when one wants to define the surgery procedure. The latter one is useful to prove the existence of necks when a singularity is approached. Clearly, a geometric neck is also a curvature neck. Conversely, Hamilton proves that a curvature neck is locally a geometric neck, that is, that one can detect a neck from the curvature alone.

In our context where $\mathcal{M}$ is an immersed manifold, it is natural to consider notions of neck which also take into account the extrinsic curvature. We say that a geometric neck is a hypersurface neck if the Weingarten operator of the cylinder is $\varepsilon$-close at every point to the one induced by the parametrization. 
A useful feature of Hamilton's definition of geometric neck (which also holds for hypersurface necks) is that the condition of being close to a cylinder is only local. In this way, the axis or the radius of the cylinder is allowed to vary, provided they do it slow enough so that every point has a neighborhood of given radius sufficiently close to a cylinder. For instance, a sufficiently thin torus is an $\varepsilon$-hypersurface neck. Also, we can have a long neck with quite different radii in the different parts. For instance, it may have in the central part a radius smaller by a factor, say, 10 than the radius at the ends. This is the kind of necks we want to remove by surgery, because in this way we reduce the curvature of the surface in that region by the same factor. Another important property enjoyed by geometric necks is that, if two of them overlap, then their union is again a geometric neck. Thus, every $\varepsilon$-neck has a maximal extension.

Let us now describe explicitly our surgery procedure. Suppose that we have an $\varepsilon$-hypersurface neck $N: S^{n-1} \times[a, b] \rightarrow \mathcal{M}$, with $\varepsilon$ small enough and $b-a$ large enough. We denote by $(\omega, z) \in S^{n-1} \times[a, b]$ the coordinates in the neck. Let us choose $\tau>0$ suitably small and $\Lambda, B>0$ suitably large. We are going to replace smoothly the image of $S^{n-1} \times[a, b]$ under $N$ by two appropriate regions diffeomorphic to disks. We only describe the region attached to the first end $z=a$, since the other one is symmetric. First let us denote by $\bar{C}_{a}: S^{n-1} \times \mathbb{R} \rightarrow \mathbb{R}^{n+1}$ the straight cylinder best approximating $\mathcal{M}$ at the cross section $z=a$. Then the standard surgery with parameters $\tau, \Lambda, B$ is performed as follows.

(i) In the region corresponding to $z \in[a, a+2 \Lambda]$, we bend the surface inwards replacing the original parametrization $N$ by

$$
\tilde{N}(\omega, z):=N(\omega, z)-\tau \exp \left(-\frac{B}{z-a}\right) \nu(\omega, z) .
$$

It can be shown that, if the parameter $\varepsilon$ measuring the quality of the neck is small enough, and if the surgery parameters $\tau, \Lambda, B$ are chosen appropriately, then the deformed surface is strictly convex in the part with $z \in[a+\Lambda, a+2 \Lambda]$.

(ii) To blend the resulting surface into an axially symmetric one we choose a fixed smooth transition function $\varphi:[0,3 \Lambda] \rightarrow \mathbb{R}^{+}$with $\varphi=1$ on $[0, \Lambda], \varphi=0$ on $[2 \Lambda, 3 \Lambda]$ with $\varphi^{\prime} \leq 0$. We then define, for $z \in[a+\Lambda, a+2 \Lambda]$

$$
\hat{N}(\omega, z):=\varphi(z) \tilde{N}(\omega, z)+(1-\varphi(z)) \tilde{C}_{a}(\omega, z) .
$$

where $\tilde{C}_{a}$ is obtained from the cylinder $\bar{C}_{a}$ by applying the bending defined in (i).

(iii) We finally modify the radius of $\tilde{C}_{a}$ for $z \in[a+2 \Lambda, a+3 \Lambda]$ in such a way that it tends to 0 as $z \rightarrow a+3 \Lambda$ and that $\tilde{C}_{a}\left(S^{n-1} \times[a+\right.$ $2 \Lambda, a+3 \Lambda])$ is a smooth axially symmetric convex cap. We do not need to write an explicit expression here because knowing that the 
cap is convex and independent of the original surface is sufficient for the estimates.

It is now possible to prove that, if $\varepsilon$ is small enough and if the surgery parameters are chosen appropriately, the estimates of Theorems 5.1, 6.1 and 6.3 remain valid for a flow with surgeries. Such a property is not surprising: bending inwards an almost cylindrical region and closing it with a convex cap should not decrease the convexity of the surface, nor produce parts with large gradients of the curvature or affect the cylindrical estimates. However, the proofs are far from being trivial and require careful computations, see [29]. Actually, in [29], the theorems recalled above are proved directly in the case of a flow with surgeries, to show more clearly that they hold also in this case.

\section{Neck detection}

After having described the surgery procedure, we have to show that it allows one to define a flow after the singularities until the surface is split into components with known topology. As a first step, we need results ensuring that, as the singular time is approached, either we can find a neck on our surface or we can tell that the surface is convex so that its topology is known. We discuss such results in this section.

We first introduce some notation. Given $p \in \mathcal{M}, t, r, \theta>0$, with $\theta \leq t$, the backward parabolic neighborhood centered at $(p, t)$ is the set

$$
\mathcal{P}(p, t, r, \theta)=\left\{(q, s): q \in d_{t}(p, q) \leq r, s \in[t-\theta, t]\right\},
$$

where $d_{t}$ denotes the distance on $\mathcal{M}$ at time $t$. If we consider a flow with surgeries, the above set may be not well defined. In fact, the $r$-neighborhood of $p$ at time $t$ may intersect a region which has been inserted with a surgery at some time between $t-\theta$ and $t$. If this happens, we say that the backward parabolic neighborhood contains surgeries.

The next result is an essential tool to prove the existence of necks before a singularity. We shall call it in the following the neck detection theorem.

THEOREM 8.1. Let $\mathcal{M}_{t}, t \in[0, T[$ be a mean curvature flow with surgeries starting from an immersed manifold $\mathcal{M}_{0}$ which is closed, two-convex and with dimension $n \geq 3$. Let $\varepsilon, \theta, L>0$ be given. Then we can find $\eta_{0}, H_{0}$ with the following property. Suppose that $p_{0} \in \mathcal{M}$ and $t_{0} \in[0, T[$ are such that

(ND1) $H\left(p_{0}, t_{0}\right) \geq H_{0}, \quad \frac{\lambda_{1}\left(p_{0}, t_{0}\right)}{H\left(p_{0}, t_{0}\right)} \leq \eta_{0}$,

(ND2) the neighborhood $\mathcal{P}\left(p_{0}, t_{0}, \frac{L}{H\left(p_{0}, t_{0}\right)}, \frac{\theta}{H^{2}\left(p_{0}, t_{0}\right)}\right)$ does not contain surgeries. 
Then, for any $t \in\left[t_{0}-\theta / H^{2}\left(p_{0}, t_{0}\right), t_{0}\right]$, the ball centered at $p_{0}$ of radius $L / 2 H\left(p_{0}, t_{0}\right)$ is contained in an $\varepsilon$-hypersurface neck. The constants $\eta_{0}, H_{0}$ only depend on $\mathcal{M}_{0}$ and on $\varepsilon, L, \theta$.

Proof. We use a contradiction argument based on a rescaling procedure like the ones which are often used by Hamilton and Perelman for the Ricci flow $[\mathbf{2 3}, \mathbf{3 4}]$. Let us assume that the assertion is false. Then we can find a sequence $\left(p_{n}, t_{n}\right)$ such that $H\left(p_{n}, t_{n}\right) \rightarrow+\infty, \lim \sup \lambda_{1}\left(p_{n}, t_{n}\right) /$ $H\left(p_{n}, t_{n}\right) \leq 0$, the parabolic neighborhoods do not contain surgeries, but the points do not lie on an $\varepsilon$-neck. A contradiction will be proved if we show that a subsequence of the parabolic neighbourhoods (after rescaling) converge to the flow of a portion of the standard cylinder; in fact, this will imply that they satisfy the conclusion of the theorem for $n$ large enough.

We first perform a parabolic rescaling of the neighborhoods by a factor $H\left(p_{n}, t_{n}\right)$ and then translate space and time so that $\left(p_{n}, t_{n}\right)$ becomes $(0,0)$; in such a way, they all become flows defined in the time interval $[-\theta, 0]$ and satisfying $H(0,0)=1$.

To obtain compactness of a sequence of flows we need uniform curvature bounds. We exploit our gradient estimates (6.4) and (6.9) to obtain that $H \leq 2$ in a possibly smaller parabolic neighborhood around $(0,0)$ for every element of the sequence. In this smaller neighborhood we have therefore convergence of a subsequence to a limit flow $\tilde{\mathcal{M}}_{t}$. When we pass to the limit, the constant terms in the estimates of Theorem 5.2 and 6.1 disappear. Therefore the limit flow $\tilde{\mathcal{M}}_{t}$ is convex and satisfies $\tilde{\lambda}_{1}(0,0)=0$. Hence, it is not strictly convex at the final time. By Hamilton's strong maximum principle for tensors, it must satisfy $\tilde{\lambda}_{1}(0,0)=0$ everywhere. By the cylindrical estimates, the other curvatures coincide at each point. Then it is easy to show that the flow is a portion of a shrinking cylinder.

So far we have only proved convergence of a smaller neighborhood around $(0,0)$. However, since we have proved that $H \leq 1$ everywhere in this neighborhood, we can apply again the gradient estimates to find that $H \leq 2$ in a larger neighborhood, and prove convergence to a cylinder there too. After a finite number of iterations, we prove convergence to a cylinder of the whole original neighborhoods.

The next result deals with the case of a point where the curvature is large, but $\lambda_{1} / H$ is not small. We give the statement for a stationary surface, since the property is not related to mean curvature flow.

THEOREM 8.2. Let $\mathcal{M}$ be an immersed hypersurface satisfying the gradient estimate (6.4). Let $\eta_{0}, H_{0}$ be given. Then there exists $\gamma_{0}>1$ with the following property. Let $p \in \mathcal{M}$ be any point such that $H(p) \geq \gamma_{0} H_{0}$ and $\lambda_{1}(p)>\eta_{0} H(p)$. Then either $\mathcal{M}$ is closed and convex or there exists a point $p^{\prime} \in \mathcal{M}$ such that

$$
\lambda_{1}\left(p^{\prime}\right) \leq \eta_{0} H\left(p^{\prime}\right), \quad H\left(p^{\prime}\right) \geq H_{0}
$$

and such that $H(q) \geq H_{0}$ at all points $q$ with $d(p, q) \leq d\left(p, p^{\prime}\right)$. 
Proof. We first use the gradient estimate to show that the curvature cannot decay too fast as we move away from $p$. Then an elementary computation shows that if $\lambda_{1} \geq H$ in a large enough ball around $p$, then $\mathcal{M}$ must be convex. It follows that the only other possibility is the existence of a point $p^{\prime}$ not too far from $p$ and with curvature not too much smaller than $H(p)$, which has the claimed properties (see [29, Theorem 7.14] for the details).

The two previous theorems can be combined to prove the existence of necks before the first singular time. In fact, let $\eta_{0}, H_{0}$ be the values given from Theorem 8.1 for some choices of $\varepsilon, \theta, L$, and let $\gamma_{0}$ be the value associated to $\eta_{0}, H_{0}$ by Theorem 8.2. Then let us pick a time $t_{0}$ close to the singular time so that there exists a point $p_{0}$ such that $H\left(p_{0}, t_{0}\right) \geq \gamma_{0} H_{0}$. If $\lambda_{1}\left(p_{0}, t_{0}\right) \leq$ $\eta_{0} H\left(p_{0}, t_{0}\right)$ we can directly apply Theorem 8.1 to conclude that $p_{0}$ lies on a neck. Otherwise we apply Theorem 8.2 to find another point $p_{1}$ where Theorem 8.1 can be applied. If no such point exists, then Theorem 8.2 implies that our surface is convex and therefore diffeomorphic to a sphere; we do not need to continue the flow any longer. After the first surgery the argument is no longer so direct, because we have to ensure that hypothesis (ND2) in Theorem 8.1 is satisfied. We will see in the next section how we can deal with this difficulty.

\section{The surgery algorithm}

In this final section we provide an algorithm which determines at which time and place the surgeries are to be performed, and we show that the flow with surgeries generated by this algorithm terminates after a finite number of steps. We will fix three values $H_{1}<H_{2}<H_{3}$ suitably large. The flow defined by our algorithm will satisfy the following properties:

- the surgeries are performed at times $T_{i}$ such that $\max H\left(\cdot, T_{i}\right)=H_{3}$;

- after the surgeries are performed, we have $\max H\left(\cdot, T_{i}+\right) \leq H_{2}$;

- the regions introduced with the surgeries satisfy $H_{1} / 2 \leq H \leq 2 H_{1}$.

A flow with these properties necessarily terminates after a finite number of steps. This can be seen considering the decrease of area of the surface. The area is decreasing during the smooth evolution, by Lemma 3.1-(ii). Since the surgeries are performed on necks with approximately fixed curvature, each of them decreases the area at least by a given fixed amount. Therefore, there can be only a finite number of them.

In order to define a flow with the above properties, it is fundamental to show that we can use surgeries to decrease the curvature of our surface by a fixed factor. This will be a direct consequence of the next result.

THEOREM 9.1. Let $\mathcal{M}_{0}$ be a 2-convex closed hypersurface of dimension $n \geq 3$. It is possible to define $\eta_{1}, H_{1}$ with the following properties. Suppose that $\mathcal{M}_{t}$, with $t \in\left[0, t_{0}\right]$, is a mean curvature flow with surgeries starting 
from $\mathcal{M}_{0}$. Suppose that all the regions inserted in the surgeries have curvature less than $2 H_{1}$. Let $p_{0}$ be such that

$$
H\left(p_{0}, t_{0}\right) \geq 10 H_{1}, \quad \lambda_{1}\left(p_{0}, t_{0}\right) \leq \eta_{1} H\left(p_{0}, t_{0}\right) .
$$

Then $\left(p_{0}, t_{0}\right)$ lies on an $\varepsilon_{0}$-hypersurface neck $\mathcal{N}_{0}$, which either covers the whole component of $\mathcal{M}_{t_{0}}$ including $p_{0}$, or can be continued in each direction until one of the two following properties hold:

(i) the mean curvature has decreased to $\mathrm{H}_{1}$, or

(ii) the neck ends with a convex cap.

Proof. The precise proof of this statement is one of the longest and most technical results in [29]. We explain here in an intuitive way some of the arguments employed for this result.

Let us first consider the case of the first surgery time. We choose $\eta_{1}, H_{1}$ as in the neck detection Theorem 8.1. Then we have that $\left(p_{0}, t_{0}\right)$ lies on an $\varepsilon_{0}$-hypersurface neck, because condition (ND2) is trivially satisfied. Now we extend the neck in both directions in a maximal way. A first possibility is that the neck never ends, that is, the two ends meet, showing that the component of the surface containing $p_{0}$ is diffeomorphic to a torus $S^{n-1} \times S^{1}$. Otherwise, the neck ends somewhere. In this case, we deduce that the points in the final part of the neck do not satisfy hypothesis (ND1) of the neck detection theorem. One possibility is that the curvature is no longer large; then we have proved case (i) of the theorem. The other possibility is that $\lambda_{1}$ is no longer small; if this happens on a large enough region, then the surface must close as a convex cap, as in case (ii). To show this rigorously, a delicate argument is needed, see [29, Theorem 8.2]; in particular, it is necessary to make a more restrictive choice of the parameters $\eta_{1}, H_{1}$ than the one needed to apply Theorem 8.1 at the beginning.

Let us now complete the argument to include the case where there have already been surgeries. We consider again our starting point $\left(p_{0}, t_{0}\right)$. It is possible to show [29, Lemma 7.2] that, if the parameters have been chosen appropriately, assumption (ND2) holds at $\left(p_{0}, t_{0}\right)$ because of the gradient estimates. In fact, we are assuming that all regions inserted by the surgeries have curvature less than $2 H_{1}$, while we have $H\left(p_{0}, t_{0}\right) \geq 10 H_{1}$. Roughly speaking, if (ND2) were violated, there would not be enough time from the last surgery to $t_{0}$ to let the curvature increase from $2 H_{1}$ to $10 H_{1}$. It is important in this argument that our gradient estimates are not obtained by arguments employing interior parabolic regularity, and therefore they hold with the same constants regardless how close we are to the surgery times. This shows that also in this case $\left(p_{0}, t_{0}\right)$ possesses a backward parabolic neighborhood which is surgery-free and we can apply Theorem 8.1 to say that $p_{0}$ lies on a neck $\mathcal{N}_{0}$.

As before, we consider the maximal extension of the neck $\mathcal{N}_{0}$ and we argue that where the neck ends one of the assumptions of Theorem 8.1 must fail. Let us consider the last point $p_{1}$ where it is possible to apply 
Theorem 8.1. If the points after $p_{1}$ violate condition (ND1), then we argue as in the case without surgeries described before. Let us consider instead the case that (ND2) is violated. Observe that the curvature at $p_{1}$ may be comparable with $H_{1}$ and thus we cannot ensure the validity of (ND2) as in the case of the point $p_{0}$. However, we claim that if (ND2) is violated at the points after $p_{1}$, we are still able to describe the topology of the region, and to conclude that the neck ends with a convex cap. In fact, if $p_{1}$ is the last point where (ND2) holds, this means that the corresponding backward parabolic neighborhood is a neck at every fixed time, and it intersects on the boundary at some previous time $t_{1}$ the region inserted by a surgery. By our construction, the region inserted in a surgery starts out cylindrical and bends gradually until it closes with a convex cap. It is then possible to prove that the cylindrical part of the region must coincide with the last part of the neck $\mathcal{N}_{0}\left[\mathbf{2 9}\right.$, Lemma 7.12]. This shows that the neck $\mathcal{N}_{0}$ ends with a convex cap also in this case.

We now consider the values $\eta_{1}, H_{1}$ given by Theorem 9.1, and take the associated $\gamma_{0}$ as in Theorem 8.2. We set

$$
H_{2}=10 \gamma_{0} H_{1}, \quad H_{3}=10 H_{2} .
$$

We then define our surgery algorithm as follows. We stop the flow every time we reach a time $T_{i}$ such that $H_{\max }\left(T_{i}\right)=H_{3}$. If some connected component of the hypersurface has become convex everywhere, we neglect it. In the remaining components, we operate surgeries in order to remove all points with curvature greater than $\mathrm{H}_{2}$. To do this, let $p$ be any point such that $H\left(p, T_{i}\right) \geq H_{2}$.

If $\lambda_{1}\left(p, T_{i}\right) \leq \eta_{1} H\left(p, T_{i}\right)$, we apply Theorem 9.1 to find that $p$ lies on a neck $\mathcal{N}_{0}$ having one of the behaviors described there. If the neck covers a whole component of the surface, we know that the component is diffeomorphic to $S^{n-1} \times S^{1}$, and we neglect it. If in both directions of the neck we find points with mean curvature approximately $H_{1}$, we perform surgeries to remove the part of the neck in between, which includes the point $p$. If we find on one side points with curvature $H_{1}$ and on the other side a convex cap, then we do surgery only on one side and neglect the rest of the neck together with the cap, since this leaves the topology of the surface unchanged. If the neck ends with a convex cap in both directions, we neglect the component because it is diffeomorphic to a sphere. In all cases, the point $p$ is removed and the possible surgeries are performed on a part of the neck with curvature close to $H_{1}$.

The other case is that $\lambda_{1}(p)>\eta_{1} H(p)$ at time $T_{i}$. If the component containing $p$ is convex, it can be neglected. Otherwise, by Theorem 8.2, there is another point $p^{\prime}$ such that $\lambda_{1}\left(p^{\prime}\right) \leq \eta_{1} H\left(p^{\prime}\right)$ and $H\left(p^{\prime}\right) \geq H(p) / \gamma_{0} \geq$ $H_{2} / \gamma_{0}=10 H_{1}$. Then we apply Theorem 9.1 to the point $p^{\prime}$ as in the former case. Using the fact that $H(q) \geq H(p) / \gamma_{0}$ at all $q$ such that $d(p, q) \leq d\left(p, p^{\prime}\right)$ we can show that the neck containing $p^{\prime}$ necessarily ends on one side with 
a convex cap containing $p$. Hence the procedure described in the previous case removes the point $p$ together with $p^{\prime}$.

We then iterate the procedure until every point with curvature greater than $H_{2}$ is removed. This requires at most a finite number of surgeries, since each surgery decreases the surface area by a fixed amount. After all such points have been removed, we restart the flow. We repeat the procedure until there are no more components left. In this way we have defined a surgery algorithm with the required properties, and the proof of the main Theorem 1.1 is complete.

\section{References}

[1] U. Abresch, J. LAnger, The normalized curve shortening flow and homothetic solutions, J. Differential Geom. 23 (1986), 175-196.

[2] S.J. Altschuler, S.B. Angenent, Y. Giga, Mean curvature flow through singularities for surfaces of rotation, J. Geom. Analysis 5 (1995), 293-358.

[3] B. Andrews, Contraction of convex hypersurfaces in Euclidean space, Calc. Var. 2 (1994), 151-171.

[4] B. Andrews, Pinching estimates and motion of hypersurfaces by curvature functions, preprint (2004).

[5] S.B. Angenent, Shrinking doughnuts in "Nonlinear diffusion equations and their equilibrium states", (1989, Gregynog), Birkhäuser, Boston (1992).

[6] S.B. Angenent, J.J.L. Velazquez, Degenerate neckpinches in mean curvature flow, J. Reine Angew. Math. 482 (1997), 15-66.

[7] K.A. Brakke, The motion of a surface by its mean curvature. Princeton University Press, Princeton (1978).

[8] Y.G. Chen, Y. Giga, S. Goto, Uniqueness and existence of viscosity solutions of generalized mean curvature flow equation, J. Differential Geom. 33 (1991), 749-786.

[9] T. Colding, B. Kleiner, Singularity structure in mean curvature flow of meanconvex sets, Electron. Res. Announc. Amer. Math. Soc. 9 (2003), 121-124 (electronic).

[10] T. Colding, W.P. Minicozzi, Sharp estimates for mean curvature flow of graphs, J. Reine Angew. Math. 574 (2004), 187-195.

[11] K. ECKER, Regularity theory for mean curvature flow. Birkhäuser, Boston (2004).

[12] K. Ecker, G. Huisken, Interior estimates for hypersurfaces moving by mean curvature, Invent. Math. 105 (1991), 547-569.

[13] J. Eells, J.H. SAmpson, Harmonic mappings of Riemannian manifolds, Amer. J. Math. 86 (1964), 109-160.

[14] L.C. Evans, J. Spruck, Motion of level sets by mean curvature, I, J. Differential Geom. 33 (1991), 635-681.

[15] L.C. Evans, J. Spruck, Motion of level sets by mean curvature, II, Trans. Amer. Math. Soc. 330 (1992), 321-332.

[16] A.M. Fraser, Minimal disks and two-convex hypersurfaces, Amer. J. Math. 124 (2002), 483-493.

[17] M. Gage, R.S. Hamilton, The heat equation shrinking convex plane curves, J. Differential Geom. 23 (1986), 69-96.

[18] M.A. Grayson, A short note on the evolution of a surface by its mean curvature, Duke Math. J. 58 (1989), 555-558.

[19] R.S. Hamilton, Three-manifolds with positive Ricci curvature, J. Differential Geom. 17 (1982), 255-306.

[20] R.S. Hamilton, Four-manifolds with positive curvature operator, J. Differential Geom. 24 (1986), 153-179. 
[21] R.S. Hamilton, The Harnack estimate for the mean curvature flow, J. Differential Geom. 41 (1995), 215-226.

[22] R.S. Hamilton, Formation of singularities in the Ricci flow, Surveys in Diff. Geom. 2 (1995) 7-136, International Press, Boston.

[23] R.S. Hamilton, Four-manifolds with positive isotropic curvature, Comm. Anal. Geom. 5 (1997), 1-92.

[24] G. Huisken, Flow by mean curvature of convex surfaces into spheres, J. Differential Geom. 20 (1984), 237-266.

[25] G. Huisken, Asymptotic behavior for singularities of the mean curvature flow, J. Diff. Geometry 31 (1990), 285-299.

[26] G. Huisken, Local and global behavior of hypersurfaces moving by mean curvature, Proceedings of Symposia in Pure Mathematics 54 (1993), 175-191.

[27] G. Huisken, C. Sinestrari, Mean curvature flow singularities for mean convex surfaces, Calc. Variations 8 (1999), 1-14.

[28] G. Huisken, C. Sinestrari, Convexity estimates for mean curvature flow and singularities of mean convex surfaces, Acta Math., 183 (1999), 45-70.

[29] G. Huisken, C. Sinestrari, Mean curvature flow with surgeries of two-convex hypersurfaces, preprint (2006).

[30] M. Marcus, L. Lopes, Inequalities for symmetric functions and hermitian matrices, Canad. J. Math. 9 (1957), 305-312.

[31] F. Mercuri, M.H. Noronha, Low codimensional submanifolds of Euclidean space with nonnegative isotropic curvature, Trans. Amer. Math. Soc. 348 (1996), 2711-2724.

[32] J.H. Michael, L.M. Simon, Sobolev and mean value inequalities on generalized submanifolds of $\mathbb{R}^{n}$, Comm. Pure Appl. Math. 26 (1973), 361-379.

[33] W.W. MulLins Two-dimensional motion of idealised grain boundaries, J. Appl. Phys. 27 (1956), 900-904.

[34] G. Perelman, The entropy formula for the Ricci flow and its geometric applications, preprint (2002).

[35] G. Perelman, Ricci flow with surgery on three-manifolds, preprint (2003).

[36] G. Perelman, Finite extinction time for the solutions to the Ricci flow on certain three-manifolds, preprint (2003).

[37] J.-P. SHA, p-convex Riemannian manifolds, Invent. Math. 83 (1986), 437-447.

[38] X.-J. WAng, Convex solution to the mean curvature flow, preprint (2004).

[39] B. White, The nature of singularities in mean curvature flow of mean-convex sets, J. Amer. Math. Soc. 16 (2002), 123-138.

[40] H. Wu, Manifolds of partially positive curvature, Indiana Univ. Math. J. 36 (1987), $525-548$.

Dipartimento di Matematica, Università di Roma "Tor Vergata” Via della Ricerca Scientifica, 00133 Roma, Italy

E-mail address: sinestra@mat.uniroma2.it 